This item was submitted to Loughborough's Research Repository by the author.

Items in Figshare are protected by copyright, with all rights reserved, unless otherwise indicated.

\title{
Misuse of "Power" and other mechanical terms in sport and exercise science research
}

\section{PLEASE CITE THE PUBLISHED VERSION}

http://dx.doi.org/10.1519/JSC.0000000000001101

\section{PUBLISHER}

Lippincott, Williams \& Wilkins @ National Strength \& Conditioning Association

\section{VERSION}

AM (Accepted Manuscript)

\section{PUBLISHER STATEMENT}

This work is made available according to the conditions of the Creative Commons Attribution-NonCommercialNoDerivatives 4.0 International (CC BY-NC-ND 4.0) licence. Full details of this licence are available at: https://creativecommons.org/licenses/by-nc-nd/4.0/

\section{LICENCE}

CC BY-NC-ND 4.0

\section{REPOSITORY RECORD}

Winter, Edward M., Grant Abt, F.B. Carl Brookes, John H. Challis, Neil E. Fowler, Duane V. Knudson, Howard G. Knuttgen, et al.. 2019. "Misuse of "power" and Other Mechanical Terms in Sport and Exercise Science Research". figshare. https://hdl.handle.net/2134/20222. 


\title{
Misuse of "Power" and other mechanical terms in Sport and Exercise Science Research
}

\author{
Edward M. Winter ${ }^{1}$, Grant Abt ${ }^{2}$, F.B. Carl Brookes ${ }^{3}$ (retired), John H. Challis ${ }^{4}$, \\ Neil E. Fowler ${ }^{5}$, Duane V. Knudson ${ }^{6}$, Howard G. Knuttgen ${ }^{7}$, William J. Kraemer ${ }^{8}$, \\ Andrew M. Lane ${ }^{9}$, Willem van Mechelen ${ }^{10}$, R. Hugh Morton ${ }^{11}$, Robert U. Newton ${ }^{12}$, \\ Clyde Williams $^{13}$, M.R. (Fred) Yeadon ${ }^{13}$

\footnotetext{
${ }^{1}$ The Centre for Sport and Exercise Science, Sheffield Hallam University, Sheffield, UK

${ }^{2}$ Department of Sport, Health and Exercise Science, University of Hull, Hull, UK

${ }^{3}$ Department of Sports Science and Physical Activity, University of Bedfordshire, Bedford UK

${ }^{4}$ Biomechanics Laboratory, The Pennsylvania State University, University Park, USA

${ }^{5}$ Department of Exercise and Sport Science, Manchester Metropolitan University, Crewe, UK

${ }^{6}$ Department of Health and Human Performance, Texas State University, San Marcos, USA

${ }^{7}$ Department of Physical Medicine and Rehabilitation, Harvard Medical School, Boston, USA

${ }^{8}$ Department of Human Sciences, The Ohio State University, Columbus, USA

${ }^{9}$ Institute of Sport, University of Wolverhampton, Wolverhampton, UK

${ }^{10}$ Department of Public and Occupational Health, Vrije University Amsterdam, The Netherlands

${ }^{11}$ School of Sport and Exercise, Massey University, Palmerston North, New Zealand

${ }^{12}$ Health and Wellness Institute, Edith Cowan University, Joondalup, WA, Australia
} \\ ${ }^{13}$ School of Sport, Exercise and Health Sciences, Loughborough University, Loughborough, UK
}

\begin{abstract}
In spite of the Système International d'Unitès (SI) that was published in 1960, there continues to be widespread misuse of the terms and nomenclature of mechanics in descriptions of exercise performance. Misuse applies principally to failure to distinguish between mass and weight, velocity and speed, and especially the terms "work" and "power." These terms are incorrectly applied across the spectrum from high-intensity short-duration to long-duration endurance exercise. This review identifies these misapplications and proposes solutions. Solutions include adoption of the term "intensity" in descriptions and categorisations of challenge imposed on an individual as they perform exercise, followed by correct use of SI terms and units appropriate to the specific kind of exercise performed. Such adoption must occur by authors and reviewers of sport and exercise research reports to satisfy the principles and practices of science and for the field to advance.
\end{abstract}

\section{INTRODUCTION}

The French philosopher and Nobel Laureate André Gide (1869-1951) is reputed to have begun talks he gave with the following extract from his 1950 publication Autumn Leaves:

Everything's already been said, but since nobody was listening, we have to start again.

Sport and exercise science is the scientific study of factors that influence the ability to perform exercise (also known, according to circumstances, as physical activity) as well as the resulting adaptations. This study is directed principally at humans but it is also applicable to equine, canine, avian, and other animal contexts. 
Importantly, terms and nomenclature used to describe exercise should abide by the Système International d'Unités (SI) i.e. be simple, precise, and accurate. The SI system comprises seven base units, prefixes and derived units (Table 1). This enables scientists from different disciplines to communicate effectively (24) and germane here, to advance sport and exercise science. With Institutional ethics approval, the purpose of this review is to highlight principally how "power", but also other SI mechanical variables, are misused in many exercise science research reports and then indicate correct use of terms and nomenclature that best describe and evaluate exercise performance. The review will define exercise and then proceed to examine misuse of mass and weight, work, velocity, power, and efficiency. For all physical activities Newton's Second Law will be demonstrated as the fundamental mechanical relationship used to document the causes of performance. A case will be made to abandon the phrase "critical power" and adopt instead "critical intensity" for the otherwise laudable concept of tolerance to exercise. Finally, a recommendation will be made to ensure that if sport and exercise science research is to be recognised as an established and credible area of application of science and so advance, terms and nomenclature to describe the performance of exercise must abide by principles of mechanics laid down by Newton and in turn, use the SI.

Table 1: Example of units in the SI system.

\begin{tabular}{lcc}
\hline Base Quantities & Name & Symbol \\
\hline Length & meter & $\mathrm{m}$ \\
Mass & kilogram & $\mathrm{kg}$ \\
Time & second & $\mathrm{s}$ \\
Electric current & ampere & $\mathrm{A}$ \\
Thermodynamic temperature & kelvin & $\mathrm{K}$ \\
Amount of substance & mole & $\mathrm{mol}$ \\
Luminous intensity & candela & $\mathrm{cd}$ \\
\hline Some Derived Quantities & & \\
\hline Frequency & hertz & $\mathrm{Hz}$ \\
Force & newton & $\mathrm{N}$ \\
Pressure & pascal & $\mathrm{Pa}$ \\
Energy & joule & $\mathrm{J}$ \\
Power & watt & $\mathrm{W}$ \\
\hline
\end{tabular}




\section{EXERCISE}

For military, occupational, and within the last two hundred years or so, sport-, leisure-related, health and quality-of-life reasons, the need to quantify either total exercise accomplished or the effectiveness with which exercise is performed has been a principal focus. This focus continues.

The World Health Organisation defines exercise as:

A subcategory of physical activity that is planned, structured, repetitive, and purposeful in the sense that the improvement or maintenance of one or more components of physical fitness is the objective. (http://www.who.int/dietphysicalactivity/pa/en/).

Exercise can also be defined as:

A potential disruption to homeostasis by muscle activity that is either exclusively or in combination, concentric, isometric or eccentric.

Only one of these definitions (33) acknowledges that either deliberately or out of necessity, gross external movement is not always a primary outcome. Where accelerated movement does occur, the activities are dynamic. Where it does not, the activities are static. Examples of the latter are the primarily isometric muscle actions in balance, a yoga pose, or in gymnastics, strength poses such as the crucifix on rings.

In some sports such as gymnastics, and weight-lifting, movement after completion of dismount or lift is undesirable and is penalised by the judges or referees. In others such as archery and shooting, stillness is crucial for performance (34). Even in dynamic sports such as luge, skeleton bobsled and swimming, the ability to hold streamlined positions of the body is decisive (http://www.geomagic.com/en/community/case-studies/british-team-uses-geomagic3d-reverse-engineering-to-streamline-/, 9). Similarly, in sailing, the ability to maintain high-force, isometric muscle activity for prolonged durations is crucial. In scrums in Rugby Union, 16 players can be primarily exercising isometrically for $10 \mathrm{~s}$ or so with maximal effort, yet minimal external movement occurs. Even in dynamic activities such as running and swimming, stabiliser and fixator muscles act either actually or quasi isometrically. Moreover, many activities of daily living require little or no movement (e.g. maintenance of posture, supporting objects in domestic tasks, screwing the tops on jars until tight and maintaining yoga poses).

While the ability of muscle to exert force in a discrete task is important, the ability repeatedly to exert force (i.e. sustain exercise in endurance activities), is equally important. Effective endurance performance requires an ability to delay the onset of fatigue - taken here to be "any reduction in force-generating capacity (measured as maximum voluntary muscle action), regardless of the task performed" (5). 


\section{QUANTIFYING THE ABILITY TO PERFORM EXERCISE}

Precise quantification of exercise is an integral part of research to improve our knowledge and understanding of factors that influence the ability to perform exercise. However, there is a key confounding factor that traps the unwary: human and other animal bodies are not simple, rigid systems. They are complex, multi-segment systems and muscular performance does not always result in movement. Even where movement does occur and in spite of concerns expressed by many $(1,17,18$, $24,27,30,33)$, exercise science researchers frequently misapply classical mechanics presented by Newton in 1687 in his three-volume Philosophæ Naturalis Principia Mathematica (Mathematical Principles of Natural Philosophy). Misapplications are most common for the mechanical variables "work", "velocity", "power" and "efficiency". These terms have strict definitions in Newtonian mechanics, the SI, and exercise science $(17,24,25)$, yet frequently, they are used incorrectly. The use of incorrect, vague, and colloquial meanings of standardized mechanics terms creates numerous problems for readers and the field of exercise science. For instance, imagine a multi-disciplinary collaboration where a nutritionist, coach and sport psychology consultant want to use the same word "power" for different things when working with an athlete. The nutritionist uses power to describe the rate of transfer of chemical energy from food, the coach uses "quick power" and "long power" to describe energy systems in sport and the psychologist uses power to describe the mental energy/focus on the task at hand. How do these people communicate? How does the athlete understand them or integrate their advice with the strength and conditioning coach who talks about "power output" in sport? The answer to these questions is simple: "With great difficulty and not according to the principles of science".

Abuses also include use of "workload" $(18,31,33)$ and "work rate" (24). Moreover, the important and highly relevant impulse-momentum relationship that expresses Newton's second law is frequently overlooked. In spite of the publication in 1960 of the SI that was intended to standardise terms, units and nomenclature, there continue to be misapplications, irregularities and transgressions in expression in exercise science research`. These include failures to distinguish between variables as basic as mass and weight.

\section{MASS AND WEIGHT}

Mass is the amount of matter in a body. The unit in which this amount is quantified and expressed is the kilogram $(\mathrm{kg})$. Weight is the force that results from the action of a gravitational field on a mass (24). It is expressed in the eponymous unit, the newton, named after Sir Isaac Newton. The symbol is $\mathrm{N}$.

If body weight is reported, it should be expressed in newtons. Yet, frequently in high-ranking journals, even those that have "science" in their title, published manuscripts allow expression of body weight in kg. Similarly, in friction-braked cycle ergometry, external resistance is sometimes expressed in $\mathrm{kg}$ or as a percentage of body mass. In both instances, this is simply incorrect, because since resistance is a force, it should be expressed in $\mathrm{N}$ or as a percentage of body weight. Use of the term "resistance" in strength and conditioning usually implies gravitational resistance, although elasticity of tissues and structures could also be involved, so the direction (vertical) required of a vector quantity like force is accounted for. 


\section{MECHANICAL WORK AND POWER}

For dynamic activities, mechanical work is what is done when:

A force moves its point of application such that some resolved part of the displacement lies along the line of action of that force.

The unit in which work is expressed is eponymous, the joule, named after the physicist and English brewer James Prescott Joule (1818-1889). It is an $\mathrm{SI}$ derived unit, has the symbol $\mathrm{J}$ and is defined as what is done when:

A force of one newton moves through a distance of one metre.

Work is usually calculated as $\mathrm{N} \cdot \mathrm{m}$.

Power is defined as:

The rate of performing work.

The unit is also eponymous: the watt, symbol W. It is named after the Scottish mechanical engineer James Watt (1736-1819). It should be made correctly as a mean value for some duration, although instantaneous power flows can be calculated. However, power flows so calculated can vary widely and are strongly influenced by the model and data used to calculate power (17). If interpretation is to be meaningful, selection of duration must be made with care.

Similar to time (s), speed $\left(\mathrm{m} \cdot \mathrm{s}^{-1}\right)$, and temperature $(\mathrm{K})$, both work $(\mathrm{J})$ and power (W) are scalar quantities. Scalars possess magnitude but not direction, as opposed to vector quantities such as velocity, force and change of temperature that possess both. The use of the term "power" in exercise science research reports should be used correctly, so the context must satisfy its strict requirements and be appropriate to documenting performance. For example, in cycle ergometry, exercise science research reports should refer to the mean external power output. This is because the ergometer does not measure the energy used to accelerate the performer's limbs or the energy wasted in impulses applied to the pedals in non-propulsive directions.

In exercise, forces are exerted by skeletal muscles that create moments of force which tend to rotate joints (23). The function of skeletal and other types of muscle is to exert force, and they do so by attempting to shorten. If the attempt is successful, concentric muscle activity occurs. If the overall muscle-tendon unit remains the same length, the activity is said to be isometric. When muscle is lengthened while it is exerting force, the action is called eccentric. Swammerdam's experiment some 300 years ago, cited in Needham (22), demonstrated clearly that when active, muscle does not decrease in volume. Hence, and as Rodgers and Cavanagh (24) indicated, the expression "muscle contraction" is simply wrong and at best inexact; it is not scientific. Cavanagh (6) therefore advocated that the phrase "muscle action" is the most accurate term for use in exercise science.

For muscle to exert force, chemical energy is required. Principally, this is supplied from forms of carbohydrate, fat, and protein but metabolism and accompanying biochemical reactions release the energy that allows muscle to 
function. The currency of this energy is adenosine triphosphate (ATP) and related high-energy phosphagens. The challenge during exercise is to meet required energy demands and so synthesise and re-synthesise ATP.

Against this brief background, consideration can now be given to correct the erroneous use of scalar and vector mechanical variables to describe exercise performance.

\section{SIMPLE MEASURES}

The simplest forms in which exercise can be quantified are distance $(\mathrm{m})$ and time (s) required for movement. In running events, overall performance is often accurately described by time. These types of event could also be investigated by converting this time and distance information into the scalar quantity speed. Speed though, is not synonymous with velocity. In a $10,000 \mathrm{~m}$ race on a 400-m track the mean velocity is zero since athletes finish where they started. The same applies in swimming in 50-m pools for events such as $100 \mathrm{~m}, 200 \mathrm{~m}$ and $1500 \mathrm{~m}$.

If performance is to be expressed as work, there must be some measureable and meaningful quantification of joules produced. For example, this cannot occur in isometric muscle activity where no notable body movement occurs. Similarly, when activities are recorded as distances covered by players in field games such as Association Football, codes of rugby, and court-based games, the use of "joules" cannot occur. Nevertheless, these types of activity can and often do require considerable expenditures of energy.

\section{THE IMPULSE-MOMENTUM RELATIONSHIP}

This relationship is fundamental to all activities in sport and exercise because it is Newton's Second Law. The Principia stated, although the original was in Latin:

The change of momentum of a body is proportional to the impulse impressed on the body, and happens along the straight line on which the impulse is impressed.

This law of motion, so expressed or in the instantaneous version ( $\Sigma \mathrm{F}=\mathrm{ma}$ where $\mathrm{m}$ is the system mass and a centre of mass acceleration) documents the mechanistic cause-effect of how forces modify motion. The vector nature of forces, impulses, acceleration, and momentum means that these calculations are performed in defined directions relevant to documenting the motion.

The law can be expressed mathematically as follows (33):

$F \propto a$

where: $F$ is the mean force and $a$ is the resulting mean acceleration.

By introducing a constant, $m$, the proportionality expression can be changed into an equation:

$F=m \cdot a$

where: $F$ is mean net force and $m$ is the mass of an object. 
Acceleration, $a$, is the rate of change of velocity so the equation can be expressed as:

$F=m \cdot((v-u) / t)$

where: $v$ is final velocity, $u$ is initial velocity and $t$ is the duration over which the change occurs. This can be rearranged to:

$F \cdot t=m \cdot v-m \cdot u$

where: $F \cdot t$ is the impulse of the force and $m \cdot v-m \cdot u$ is the change of momentum of the body, hence the name: the impulse-momentum relationship.

For an activity such as vertical jumping in which initial velocity, $u$, is 0 , the expression becomes:

$F t=m \cdot v$

In a vertical jump, there is a, vertical reaction force, $R$, that acts upwards and a weight, $\mathrm{mg}$, that acts vertically downwards. In the above formula, the net force $F,=R-m g$.

Rearrangement of the equation allows the velocity of the body at departure or release to be identified:

$(F \cdot t) / m=v$

This relationship is precise, mathematically irrefutable and describes not only requirements for performance but importantly, also explains pre-requisites for performance.

For projectile activities in which an object is thrown, kicked, struck with an implement such as a racket or stick, or when the projectile is the body as in horizontal and vertical jumping, it is the velocity of the mass centre at departure or release and the mass centre location in space that determine trajectory (1). The vector nature of velocity documents both magnitude (speed) and direction of the object's initial motion

Hence, the object could be propelled at great speed or alternatively, at low speed with delicacy as for instance a drop-shot in racket-sports. Neither high nor low speed is effective without accurate direction. It is the impulse applied to the object by the performer either directly or with the assistance of an implement that enables the performer to defeat their opponent. In these cases, claims that a racket or performer is powerful are misuses of terms. In fact, the performer or racket may be said to be impulsive.

Effective technique requires the integration of several factors so as to optimise impulse in the appropriate timing and direction for a movement task. For example, large forces are required but if they are too large, injury to muscle or tendon and in extreme cases, bone, could occur (12). When optimising throwing technique to maximise distance thrown in events such as shot-put, discus and javelin, the duration of contact with the implement before its departure is an important measure. Similarly in jumping, techniques are designed to capitalise on duration of contact with the ground immediately before departure into the air (3). These durations must provide a compromise of numerous factors including the jump goal, preparatory 
motions, and exploitation of neuro-muscular properties using eccentric-to-concentric stretch-shortening cycle muscle actions (19).

The ability to develop impulse is also important in field games such as rugby, association football, and field- and ice-hockey as well as court-based games such as tennis, squash, and basketball. Players either have to outwit opponents with swerves or "cuts" (side-steps) or change direction rapidly to reach a ball or avoid a tackle. Such movements require changes in velocity i.e. where both speed and direction are deliberately changed. Changes in these properties are determined by a generated impulse.

The words "power" and "explosive" are ubiquitously applied in research and professional practice to tasks that are brief and require maximal neuromuscular activation such as jumps, strikes, kicks and throws, as well as weightlifting and resistance training (17). This is in part driven by the proliferation of inexpensive and easy-to-use systems to assess kinematics and kinetics during these movements, particularly in the field of strength and conditioning. Such devices produce an array of variables, some of which are measured directly and others derived based on Newtonian physics. However, they are often poorly defined, are not valid, or simply do not represent the performance being assessed. Of particular concern is use of the word "explosive". This is not a physics term and of course nothing actually "explodes" in the human. We recommend that the term "explosive" no longer be used to describe human movement.

"Power" is often expressed as a "clearly defined, generic neuromuscular or athletic performance characteristic" rather than as an application of the actual mechanical definition (17) which leads to considerable inaccuracy and confusion. We reiterate that maximal neuromuscular efforts have the goal of maximising the impulse produced as this determines the resulting velocity as a result of the impulsemomentum relationship. Humans with inherent or developed abilities in such movements would be more accurately described as "highly impulsive" and the most appropriate measure of such performance is the impulse they produce. To reinforce the point, power is a scalar quantity with both peak and mean measures poorly related to jumping or throwing performance compared with resultant force or impulse that predominantly dictate the performance outcome.

So far, the focus has been on discrete actions but in many sports and activities, actions are not discrete i.e. they do not occur only once, they have to be performed repeatedly; for hours in the case of tennis and marathon running. This leads to consideration of effective impulse in endurance activities.

\section{ENDURANCE ACTIVITIES: REPEATED IMPULSES}

In endurance activities such as long-distance cycling and running, it is the ability repeatedly to generate impulse that is decisive. In cycling, force by each leg is applied that creates an angular impulse which drives the rotation of the pedals and the drive mechanism of the bicycle. In one revolution of the pedal crank, two such impulses are applied. This contrasts with four-stroke internal-combustion engines, where, for single-cylinder engines, there is only one propulsive phase for two revolutions of the crankshaft. A flywheel smooths the pulsatile impulses. Each individual impulse is applied for about only $120^{\circ}$ of crankshaft motion (28) to create an angular impulse about the crankshaft. Multi-cylinder engines reduce the pulsatile nature, so six-cylinder or greater configurations have no gaps in impulse. Race 
engines that can exceed $18,000 \mathrm{rev} \cdot \mathrm{min}^{-1}$ do not need a flywheel, because times between impulses are miniscule.

The linear impulse in cycling or in engines creates a moment of force and hence angular impulse. For convenience, performance in cycle ergometry or combustion engines is expressed by steady-state power flows from the impulses that created them. However, most human movement is dynamic, not steady state about a non-moving axis of rotation; so, external power flow is a poor descriptor of performance compared to the impulses that change velocity. For effective tangential forces in cycling, coordination of recruitment of numerous muscles has to occur to optimise innervation, elasticity of structures - principally muscle and tendon - muscle fibre types and metabolic determinants of force production. This is vital both for sprinting and prolonged cycling. As with four-stroke engines, each propulsive impulse occurs for approximately $120^{\circ}$ of crankshaft rotation. The mean torque (propulsive moment of force) or mean power output are secondary expressions of the forces that have created and modified the movement.

In running, the same logic applies. Running is a series of impulsive footstrikes with the ground and, in endurance running, the athlete's structural, innervation, and metabolic characteristics have to be optimised to maintain the ability to generate impulse so as to maximise progression. This optimisation is an exceedingly complex integration of biochemical, biomechanical, physiological, psychomotor, and other factors (7). Endurance running needs to be economic so as to use as little chemical energy as possible and similarly, minimise unproductive mechanical energy.

As Winter (30) clearly indicated, this optimization or economic production of effective forces to modify movement should not to be confused with "efficiency". Efficiency in engines is a ratio of the work output to the energy input. Efficiency applied to human movement tries to create a simple ratio of the mechanical work performed to the physiological energy expended:

\section{(External mechanical work done/energy expended) x 100}

There are, however, numerous problems with this simple ratio as an indicator of performance given the complexity both of the numerator and denominator. For running, it is virtually impossible to meaningfully calculate the numerator in this expression. So in turn, determination of a meaningful measure of efficiency is also impossible $(7,30)$. There are also problems with uniquely separating the internal mechanical energy (energy to move limbs) and the external mechanical energy. There are special issues of journals on this topic for interested readers $(2,7)$. While is it also tempting to assume the energy expended is simply the oxygen consumption measured over the event, like the numerator there is clearly more chemical energy being used by the body than is being accounted for in the denominator. Even so, misuse of "efficiency" persists (11).

In field games, the ability to repeatedly accelerate, decelerate, change direction, and, kick or strike a ball, determines effective performance. All of these actions require the ability to repetitively generate well-timed and directed impulses. That ability encompasses skill to perform the action per se and endurance to do so repeatedly. Deficiencies in one or both will adversely affect performance.

For these activities, it is common to hear said or even read in research reports of players performing supposedly at a "high work rate." If they were, by definition, 
their power output would be high. However, the assessment of external mechanical work done is not possible hence, the term "work rate" is inapplicable. It is colloquial and should not be used (24). As the expression tends to be directed at players who run large distances at high speeds, an acceptable alternative term is "high-intensity play".

In cycling, it might be convenient to assess external power output, but this construct is an approximation of the fundamental requirement: external impulse generation by the body. Moreover, selection of duration for mean power is important, since there are considerable differences between mean and instantaneous power flows. In maximal-intensity exercise, probably a mean value for at least a complete pedal revolution (32) is required and in endurance activities, probably minutes if reliable values of this secondary measure of performance are to be obtained.

\section{THE MISNOMER "CRITICAL POWER"}

In 1965, Monod and Scherrer (21) announced a laudable method to quantify an intensity of exercise that marked a limit to what was tolerable, primarily through aerobic metabolism, although it should be acknowledged that Hill (13) had outlined the principle some 40 years earlier. This intensity was theoretical and represented what could be sustained for infinite duration although in practice under laboratory conditions, typical durations are 20 - 45 minutes (16). The intensity was termed "critical power". A search on Medline (14 April 2015) revealed that, since Monod and Scherrer's (21) founding publication, 208 exercise-based manuscripts have been published that used the expression. At first sight, the term appears to be well established, academically acceptable, and attractive but closer inspection quickly reveals otherwise.

The majority of published studies (approximately two thirds) purporting to use "critical power" have used some form of cycle-ergometer task. Typically, four to six bouts of all-out cycling to volitional exhaustion are performed at different external resistances. Ideally, each bout occurs on a separate day. There is a hyperbolic relationship between on the ordinate, mean external power output measured on a cycle ergometer (using the product of external resistance and flywheel rotation to determine the distance travelled by an imaginary point on the periphery of the flywheel) and on the abscissa, duration of exercise i.e. time to exhaustion. This becomes a positive linear relationship when mean external power output is expressed as a function of the reciprocal of duration. The vertical intercept of the relationship is referred to as the "critical power". An alternative way to calculate "critical power" is to determine external mechanical work done (J) i.e. power output multiplied by duration, and relate that to duration. This too is a positive linear relationship. The slope of the regression line has also been called "critical power".

However, changes in pedalling rate affect the identified "critical power"; it is less at greater pedalling rates than at lower (4). This is explained principally by two factors. First, Hill's (14) muscle force-velocity relationship and second, additional internal mechanical work that is required to move the limbs (30). It is the latter that probably has more effect and effectively highlights the folly of the term. The lower limbs are substantial structures in that they comprise some $32 \%$ of total body mass (8). Forces exerted by muscle to accelerate and decelerate these limbs sequester energy that would otherwise be used for useful external output. Unless pedalling rates are controlled, comparisons of "critical power" and the implied optimality of this concept are compromised (4). According to Hill's force-velocity relationship in muscle (14), the optimisation of power output requires different pedalling rates for 
different external resistances. It is thus difficult to achieve overall optimisation of all factors involved. Similar force-velocity and technique variables confound the use of external power flow in jumping $(20,26)$. A scientist would ask, why abandon understanding of $100 \%$ variance using impulse-momentum to use confounded secondary measures such as power flows to study causative factors of movement?

Add to this the problems previously noted in the adequacy of external power as a secondary measure of performance and the energy/work/power not accounted for, one may conclude that exercise science literature should avoid use of the concept of "critical power". Use of the term perpetuates the erroneous assumption that a vague, colloquial meaning of "power" has a clear scientific meaning and is universally applicable in the study of exercise performance. This parallels the problems for a practitioner-understanding of muscular performance and exercise science when in the strength and conditioning literature, the term "power" is used as a surrogate for all muscular performance that includes extremes of force or speed (17).

When the concept is applied to running and swimming, performance can be expressed as mean speed. Using similar mathematical principles as for cycling, there is a positive linear relationship between distance to exhaustion on the ordinate and time to exhaustion on the abscissa. The slope of the regression line gives "critical speed". Clearly, the term "power" and hence "critical power" is inapplicable, although the term was still used in 11 manuscripts. It should also be noted that the term "critical velocity" is sometimes used (66 relevant Medline citations). Unfortunately, such use is frequently incorrect. The vector nature of velocity challenges its use, whereas use of the scalar "speed" is not so challenged. The scalar speed is preferable because it is usually the measure of interest. Moreover, the term "speed" is more likely to be understood by the athlete and his or her support team, whereas "power" could be interpreted differently, as indicated earlier.

For isometric muscle activity, mean force can be plotted against duration of force application. In this case, all the terms "work", "power" and "speed" are inapplicable. Monod and Scherrer (21) acknowledged this, albeit erroneously:

"Static contraction does not affect work in the physical sense." (page 333)

The error is because "work" is simply inapplicable; it is the wrong mechanical construct to use in this context.

Monod and Scherrer (21) were aware of this and in addition wrote:

"The critical rate of static work (sic) has the dimension of a force. Therefore it is in fact a critical force." (page 334).

\section{0. "CRITICAL INTENSITY"}

Despite its apparent popularity in the literature, the term "critical power" has limited applicability. It should be restricted to: activities where steady-state mean external power output is relevant to performance; when it can be meaningfully assessed; and when confounding factors (e.g. pedalling rate) can be controlled. The potential relevance of the term is also compromised by failure to consider the important contributions of internal power requirements that are apparent in greater cycling rates of the limbs. Instances where such assessment and control occur are rare in the exercise science literature. The term "critical speed" can be used where it 
is impossible, or at best exceptionally difficult, to get any measure of external power output. The term "critical force" may be used where isometric muscle activity is the interest because "power" is simply inapplicable.

However, "critical power", "critical speed", and "critical force" are all measures of the same quality: a critical intensity of exercise. This intensity marks a limit to what is sustainable before fatigue makes the performer slow down, or reduce force application. It is inconsistent and nonsensical to have three names for the same phenomenon. It is also incorrect to express critical power (a mechanical power) in the units of speed $\left(\mathrm{m} \cdot \mathrm{s}^{-1}\right)$, force $(\mathrm{N})$, or torque $(\mathrm{N} \cdot \mathrm{m})$. Such expression is counter to Newtonian mechanics, the $\mathrm{SI}$, and standards of scientific reporting. Together, the several terms and non-compliance with Newton are quite simply, not science. Monod and Scherrer (21) identified this failing, but seemed unsure how to rectify matters. Some 50 years on, the solution is remarkably simple: the term "critical power" should be replaced with "critical intensity" and documented with the appropriate SI units depending on the particular movement or action.

The ability to tolerate exercise at high intensity for long durations is the key characteristic of successful endurance athletes. Importantly, this tolerance embraces statics that is relevant to many activities and sports such as gymnastics, climbing, cycling, swimming, and running.

\section{APPROPRIATENESS OF "INTENSITY"}

Use of "intensity" to express the challenge posed by exercise was first advocated by Knuttgen (18). It is an elegant way to avoid misuse of mechanical constructs. Objections to use of the term are unfounded. Intensity is in general use in the categorisation of exercise into domains that are based on physiological responses. Intensity domains are "moderate", "heavy", "very heavy" and "severe" (29) and "extreme" (15). These categorisations apply to all forms of static and dynamic exercise. The term is also used in the tripartite requirement for effective training i.e. frequency, intensity and duration of training. Moreover, recent interest in high-intensity interval training (10) further indicates support for acceptability and use of the term.

The term "intensity" is recognised by the $\mathrm{SI}$, but not defined universally. It is expressed as $\mathrm{W} \cdot \mathrm{m}^{-2}$. However, a principal and established use of the term is in luminescence to quantify brightness of light. The SI unit of luminous intensity is the candela, i.e. power emitted by a light source in a particular direction. It has the unit $\mathrm{cd}$, roughly equivalent to the light emitted by a candle. However, the unit is not expressed as $\mathrm{W} \cdot \mathrm{m}^{-2}$ although it could be considered to be traceable to the watt because of its definition: the luminous intensity, in a given direction, of a source that emits monochromatic radiation of a frequency $540 \times 10^{12}$ hertz and that has a radiant intensity in that direction of 1/683 watt per steradian. Moreover, another unit of light is the lumen. This is a measure of luminous flux as opposed to radiant flux. The former reflects the varying sensitivity of the human eye to different wavelengths of light whereas the latter indicates power of all electromagnetic waves emitted, independent of the eye's ability to perceive them. It is equivalent to $1 \mathrm{~cd} \cdot \mathrm{sr}^{-1}$.

While exercise could be perceived as a rate of movement through space i.e. $\mathrm{W} \cdot \mathrm{m}^{-2}$, that would not permit application to isometric activity or the scalar speed. As science develops in response to phenomena that emerge, either new units have to be developed or old ones have to be adapted. The (Shorter) Oxford English Dictionary defines intensity in physics as: "A (measurable) amount of energy, 
brightness, magnetic field etc". The "etc." is important. The term "intensity" has a utility that allows it to be applied to exercise. It avoids infatuation with "power" and other constructs and provides a solution to correct what Monod and Scherrer themselves acknowledged about "critical power": its inapplicability for isometric muscle activity and where performance is expressed as the scalar speed (21). Added to which is recognition that meaningful use of "power" is possible only if many pre-requirements are satisfied. It is rare that such satisfaction occurs.

\section{CONCLUSION}

If sport and exercise science is to advance, it must uphold the principles and practices of science. Descriptions of exercise must make correct use of basic scientific terms, nomenclature, and units. Greater recognition and use of Newton's Second Law of motion as the explanation of how forces modify movement, rather than less-accurate secondary performance variables in research reports and their critical review, are needed. Many errors in use of SI nomenclature can be rectified by adoption of the term "intensity" to categorise exercise in terms of its actual or perceived challenge and into domains based on physiological responses. While Monod and Scherrer's (21) method to identify a limit of tolerance to exercise is a valuable way to investigate mechanisms of fatigue, the self-acknowledged flaws in naming this limit "critical power" are problematic. This problem can easily be rectified: the term should be re-named "critical intensity" and performance documented by the SI units relevant to the activity being studied. Universal adoption of intensity will help reduce the confusion and perpetuation of erroneous understanding of mechanical work, energy, and power in sport and exercise. Importantly, adoption of this recommendation by journal editorial teams will help advance sport and exercise science.

\section{REFERENCES}

1. Adamson, G.T. and Whitney, R.J. Critical appraisal of jumping as a measure of human power. In: Medicine and Sport 6: Biomechanics II J. Vredenbregt and J. Wartenweiler, eds. Basel: Karger, 1971. pp. 208-211.

2. Aleshinsky, S.Y. An energy 'sources' and 'fractions' approach to the mechanical energy expenditure problem - I. Basic concepts, description of the model, analysis of a one-link system movement. J Biomech 19: 287-293, 1986.

3. Alexander, R.M. Optimum take-off techniques for high and long jumps. Philos Trans Roy Soc Ser B 329: 3-10, 1990.

4. Barker, T., Poole, D.C., Noble, M.L and Barstow, T.J. Human critical poweroxygen uptake relationship at different pedalling frequencies. Exp Physiol 91: 621-632, 2006.

5. Bigland-Ritchie, B. and Woods, J.J. Changes in muscle contractile properties and neural control during human muscular fatigue. Invited reviews in: Muscle Nerve 7: 691-699, 1984.

6. Cavanagh, P.R. On "muscle action" vs "muscle contraction." J Biomech 21: 69, 1988.

7. Cavanagh, P. R., and Kram, R. The efficiency of human movement - A statement of the problem. Med Sci Sports Exer 17: 304-308, 1985.

8. Dempster, W. T. Space requirements of the seated operator. WADC-TR-55159, Wright Patterson Air Force Base, 1955. 
9. Gatta, G., Cortesi, M., Fantozzi and Zamparo, P. Planimetric frontal area in the four swimming strokes: implications for drag, energetics and speed. Hu Mov Sci 39: 41-54, 2015.

10. Gibala, M.J., Little, J.P., Macdonald, M.J. and Hawley, J.A. Physiological adaptations to low-volume, high-intensity interval training in health and disease. J Physiol 590: 1077-1084, 2012.

11. Grassi, B., Rossiter, H.B. and Zoladz, J.A. Skeletal muscle fatigue and decreased efficiency: two sides of the same coin? Ex Sport Sci Rev 43: 75-83, 2015.

12. Heiderscheit, B.C., Hoerth, D.M., Chumanov, E.S., Swanson, S.C., Thelen, B.J. and Thelen, D.G. Identifiying the time of occurrence of a hamstring strain injury during treadmill running: a case study. Clin Biomech 20: 1072-1078, 2005.

13. Hill, A.V. The physiological basis of athletic records. Nature 116: 544-548, 1925.

14. Hill, A.V. The heat of shortening and the dynamic constants of muscle. Proc Roy Soc London B 126: 136-195, 1938.

15. Jones, A.M. and Poole, D.C. Oxygen Uptake Kinetics in Sport, Exercise and Medicine. Abingdon, UK: Routledge, 2005.

16. Jones, A.M., Vanhatalo, A.T. and Doust, J.H. (2009). Aerobic exercise performance. In: Kinanthropometry and Exercise Physiology Laboratory Manual Volume 2: Physiology, R. Eston and T. Reilly eds. Abingdon, UK: Routledge, 2009. pp. 271-306.

17. Knudson, D.V. Correcting the use of the term "power" in the strength and conditioning literature. J Strength Cond Res 23: 1902-1908, 2009.

18. Knuttgen, H.G. Force, work, power and exercise. Med Sci Sports 10: 227-228, 1978.

19. Komi, P.V. Physiological and biomechanical correlates of muscle function: Effect of muscle structure and stretch-shortening cycle on force and speed. Exerc Sport Sci Rev 12: 81-121, 1984.

20. Markovic, S., Mirkov, D.M., Nedeljkovoc, A. and Jaric, S. Body size and countermovement depth confound relationship between muscle power output and jumping performance. Hum Mov Sci 33: 203-210, 2014.

21. Monod, H. and Scherrer, J. The work capacity of a synergic muscular group. Ergonomics 8: 329-338, 1965.

22. Needham, D.M. Machina Carnis. London: Cambridge University Press, 1971.

23. Pandy, M.G. Moment arm of a muscle force. Exerc and Sport Sci Rev 27: 79118, 1999.

24. Rodgers, M. and Cavanagh, P. Glossary of biomechanical terms, concepts and units. Phys Ther 64: 1886-1902, 1984.

25. Royal Society. Quantities, Units and Symbols A Report by the Symbols Committee of the Royal Society. London: The Royal Society, 1975/1981.

26. Samozino, P., Rejc, E., di Pramprero, P.E., Belli, A. and Marin, J-P. Optimal force-velocity profile in ballistic movements-Altius: citius or fortius? Med Sci Sports Exer 44, 313-322, 2012.

27. Smith, A.J. A study of the forces on the body in athletic activities with particular reference to jumping. Doctoral thesis, University of Leeds, Leeds, UK, 1972. 
28. Smith, P.H. The Design and Tuning of Competition Engines (seventh edition revised by D. N. Wenner). Cambridge, MA: Bentley Publishers, 1977.

29. Whipp, B.J. Domains of aerobic function and their limiting factors. In: The Physiology and Pathophysiology of Exercise Tolerance. J.M. Steinacker and S.A. Ward, eds. New York: Plenum Press, 1996. pp. 83-90.

30. Winter, D.A. Biomechanics and Motor Control of Human Movement ( $3^{\text {rd }}$ edn.). Chichester, UK: Wiley, 2004.

31. Winter, E.M. Workload: time to abandon? (editorial). J Sports Sci 24: 12371238, 2006.

32. Winter, E.M., Brookes, F.B.C. and Hamley, E.J. Maximal exercise performance and lean leg volume in men and women. J Sports Sci 9: 3-13, 1991.

33. Winter, E.M. and Fowler, N. Exercise defined and quantified according to the Système International d'Unités. J Sports Sci 27: 447-460, 2009.

34. Zatsiorsky, V.M. and Aktov, A.V. Biomechanics of highly precise movements: the aiming process in air rifle shooting. J Biomech 23 Suppl 1: 35-41, 1990. 